\title{
Barreras de acceso geográfico a los servicios de salud oral en el departamento del Tolima, Colombia ${ }^{1}$ Geographical accessibility barriers to oral health services in the department of Tolima, Colombia
}

\author{
John Harold Estrada Montoya ${ }^{2 *}$ y Laura Camila Reyes Cañón ${ }^{3}$ \\ Fecha de recepción: 25 de enero de 2021 \\ Fecha de aceptación: 14 de marzo de 2021
}

\begin{abstract}
Resumen-Estudiomixtodescriptivo decortetransversal realizado para los 47 municipios del departamento del Tolima, Colombia, vecino del departamento de Cundinamarca, donde se encuentra Bogotá, la capital del país. El objetivo fue analizar las barreras de acceso geográfico y el inventario de talento humano y servicios de odontología en este departamento. Se aplicó un sistema de medida de distancia y tiempo para identificar la duración del desplazamiento desde cada municipio hasta Ibagué (capital departamental) en transporte público. El determinante de barrera de acceso geográfico avalado por la Organización Mundial de la Salud (OMS) es una duración de viaje mayor a cuatro horas. Se encontró que el primer nivel de atención en el sector público está presente en todos los municipios, el segundo nivel está en seis; el tercero únicamente en Ibagué. El desplazamiento en transporte público representa una barrera de acceso geográfico en seis municipios; la cantidad de odontólogos/as en el sector público no cubre satisfactoriamente las necesidades de la población. En el departamento del Tolima existen barreras de acceso geográfico específicamente para la salud oral.
\end{abstract}

Palabras clave: Acceso a salud, barreras de acceso geográfico, mercantilización, tiempo de viaje, Colombia.
Abstract - Cross-sectional descriptive mixed study carried out for the 47 municipalities of the department of Tolima, Colombia, neighboring the department of Cundinamarca, where Bogota, the capital of the country, is located. The objective was to analyze the geographical access barriers and the inventory of human talent and dental services in this department. A distance and time measurement system was applied to identify the duration of travel from each municipality to Ibagué (departmental capital) by public transport. The geographical accessibility barrier determinant endorsed by the World Health Organization (W.H.O.) is a trip duration greater than four hours. It was found that the first level of care in the public sector is present in all municipalities, the second level is in six; the third only in Ibague. Displacement by public transport represents a geographical access barrier in six municipalities; the number of dentists in the public sector does not satisfactorily cover the needs of the population, since more than $50 \%$ of the municipalities have a deficit of more than four general dentists and there is a low presence of specialists. In the department of Tolima there are geographical accessibility barriers to health and, specifically, to oral health.

Keywords: Access to health, geographical accessibility barriers, commodification, travel time, Colombia

\footnotetext{
${ }^{1}$ Artículo original derivado de un proyecto de investigación. Agradecemos a la Asociación Colombiana de Investigación en Odontología (IADR división Colombia), por la financiación aportada para el desarrollo de este trabajo.

2Profesor Titular de la Universidad Nacional de Colombia, Facultad de Odontología, Departamento de Salud Colectiva. Carrera 30 núm. 45-03, edificio 210, Bogotá, Colombia. ORCID: https://orcid.org/0000-0003-1135-8238?lang=es *Autor de correspondencia: calle 53 No. 37 A 66, apartamento 401, Bogotá, Colombia, zona postal: 111321. Correos electrónicos: jhestradam@unal.edu.co y jhestradam@gmail.com

30dontóloga. Estudiante de Maestría en Estudios Sociales de la Ciencia, Universidad Nacional de Colombia. Correo electrónico: Icreresc@unal.edu.co
} 


\section{Introducción}

- I Sistema General de Seguridad Social en Salud (SGSSS) colombiano regula y garantiza el acceso a servicios de salud para la población en todos los niveles de atención, bajo los principios de solidaridad, igualdad y universalidad, entre otros. Los tres niveles de atención en el país están organizados de la siguiente forma:

- Nivel I: Médico/a general o personal auxiliar o paramédico y otros/as profesionales de la salud no especializados como odontólogos/as.

- Nivel II: Médico/a general o profesional, paramédico con interconsulta, remisión o asesoría de personal o recursos especializados (como la endodoncia).

- Niveles III y IV: Médico/a especialista, con participación del médico/a general o profesional paramédico, además de cinco especialidades odontológicas (Congreso de la República, 1993).

El primero, presente en todos los municipios de Colombia, en teoría aseguraría el principio de universalidad en el acceso a los servicios de salud. La realidad empírica contrasta con los enunciados de la ley, debido a las marcadas individualidades de cada uno de los 32 departamentos que conforman Colombia. Estas diferencias se presentan en demografía, cultura, nivel socioeconómico y, sobre todo, en grandes variaciones geográficas, pues el país tiene costa en los litorales océanos Pacífico y Atlántico; lo atraviesan tres cordilleras que son la parte final de la cordillera de Los Andes, además de que posee todos los pisos térmicos y tiene una gran parte de su territorio en la región de la Amazonía, lo que resulta en que no todos los habitantes hagan efectivo el disfrute de los servicios, a pesar de que en las cabeceras municipales exista el nivel básico de atención. Lo anterior no ha permitido alcanzar las metas propuestas de aseguramiento y cobertura universales, con la implementación de la ley 100 de 1993 (Caicedo-Rosero \& Estrada-Montoya, 2016).
Este trabajo hace énfasis particular en la ubicación geográfica tanto de las personas como de las instituciones y profesionales del sector salud. Dos estudios realizados previamente por el grupo de Salud Colectiva de la Facultad de Odontología de la Universidad Nacional en dos departamentos de Colombia (Nariño y Boyacá) evidenciaron la existencia de barreras de acceso geográfico a los servicios de salud.

El uso de los referidos servicios impacta significativamente en el estado de salud de los/as habitantes de un país (Zhang, Qiu, Zhou, Zhao \& Gu, 2017; Gulliford, 2003). Es ampliamente reconocido que las personas utilizan los servicios de salud según como se presenta la ubicación y distribución de éstos (Guagliardo, 2004). El acceso a la salud se define como un concepto multifactorial con un interés creciente y se refiere a la acción de recibir la atención en salud, la búsqueda del servicio y la posibilidad que se tiene de tomarlo (Congreso de la República, 1993; Zhang et al., 2017). Cuando existe un potencial de entrega de estos servicios, pero su realización no ocurre, nos encontramos con barreras para acceder a ellos. El concepto de "acceso" involucra capacidad financiera, aspectos conductuales y la disposición de llegar al sitio donde está o donde se presta el servicio (Mauch, Kirubi, Kipruto, Sitienei \& Klinkenberg, 2011).

En este caso, una barrera se define como cualquier aspecto que interfiere y limita la utilización real de los servicios y se pueden clasificar en no espaciales y espaciales. Dentro de las primeras encontramos el género, la edad, la raza, la cultura, el lenguaje, limitaciones físicas, aspectos socioeconómicos, laborales, condiciones de salud, disponibilidad del proveedor y calidad en la atención (Caicedo-Rosero \& Estrada-Montoya, 2016; World Health Organization, 2010; Magilvy, 2000). Las segundas o geográficas varían de acuerdo con la ubicación desigual en los territorios de las instituciones y los profesionales de la salud (generales y especialistas), y con el lugar y las condiciones en que viven los/as pacientes, involucrando la distancia hasta los establecimientos 
médicos, el estado de las vías de comunicación y el tiempo de viaje requerido para llegar (partiendo de la presunción de que se dispone del dinero para hacer el viaje) (Caicedo-Rosero \& Estrada-Montoya, 2016).

Estudios realizados por la Organización Mundial de la Salud (OMS) en África (World Health Organization, 2016) revelaron que cuando la distancia entre el/la usuario/a y el/la proveedor/a de los servicios implica un tiempo de viaje en cualquier medio de transporte mayor a cuatro horas o se encuentra a una distancia mayor a 25 km, se configura una barrera de acceso geográfico; entendiendo que si se necesitan cuatro horas para ir y otras cuatro para volver, sumado al tiempo de la gestión de la atención, mayor a cuatro horas, representaría más de un día laboral para recibir el servicio (World Health Organization, 2010; Jordan, Martín \& Barnett, 2004).

En investigaciones anteriores efectuadas en los departamentos colombianos de Nariño y Boyacá se encontró que existe recurso humano insuficiente para el desempeño de actividades clínicas o de acción colectiva. Algunos municipios de estos departamentos tienen un déficit de hasta 20 odontólogos/as para atender a la población total del municipio (CaicedoRosero \& Estrada-Montoya, 2016; Estrada-Montoya \& Pulido, 2012). En Nariño, todos los municipios del área del Pacífico cuentan con el primer nivel de atención, pero no así con el segundo y el tercero, ya que se encuentran a tiempos de viaje desde seis hasta 22 horas (Ministerio de Salud y Proteccio Social, 2018).

El acceso a los servicios de salud se basa principalmente en la necesidad que se tiene de ellos, la provisión por parte de los prestadores y la utilización que la población hace de los mismos (Braveman, 2003). Esta necesidad no se expresa solamente cuando la persona está enferma, sino también cuando el padecimiento no se ha hecho evidente o para prevenirlo (Kirby \& Kaneda, 2005). Por lo anterior, la garantía de acceso a los servicios no está dada solamente por tener instalaciones apropiadas, sino que tiene que ver con su ubicación espacial, con justicia, sin importar la clase social a la que pertenece el usuario o usuaria, y el tipo de aseguramiento que tiene (Qidwai et al., 2012). De esta forma, la distancia se configura como un primer obstáculo, que se encuentra estrechamente relacionado con las otras barreras mencionadas (Comber \& Radbum, 2011).

La realización de estudios geográficos con respecto a la distribución de una enfermedad y el curso de desarrollo de la misma, que dio resultados expresados en mapas, permitieron un entendimiento del proceso salud-enfermedad en zonas determinadas, lo que estableció una relación entre la población y los factores ambientales que la rodean (Nuckols \& Jarup, 2004; Gao, Mioc, Anton, Yi \& Coleman, 2008). Los cálculos para medir la accesibilidad pueden no ser los más acertados, debido a que la medida de tiempo y distancia podría ser imprecisa, mas arrojan un resultado estimado que posibilita establecer la facilidad del acceso a un servicio de salud, incluyendo variables como velocidad y tiempo (Beyer, Saftlas, Wallis, Peek-Asa \& Rushton, 2011).

Este trabajo pretende complementar de manera positiva la anterior investigación efectuada por Caicedo-Rosero \& Estrada-Montoya en 2012 y EstradaMontoya \& Pulido en 2015, en los departamentos de Nariño y Boyacá; esta vez desde un acercamiento empírico en un medio de transporte masivo como los buses intermunicipales, lo que amplía el conocimiento y forja el camino de nuevas investigaciones en salud pública en Colombia.

\section{Materiales y métodos}

Estudio mixto descriptivo de corte transversal en 47 municipios del departamento del Tolima. El diseño metodológico se adecua a la directriz Strengthening the Reporting of Observational studies in Epidemiology (STROBE) para estudios observacionales (von Elm et al., 2008). La investigación se desarrolló en seis etapas:

\section{Búsqueda de bibliografía y selección de} fuentes de información. Se consultaron las siguientes bases de datos de uso corriente en 
Ciencias de la Salud: Pub Med, Science Direct y Medline, utilizando términos normalizados en el apartado del tesauro ${ }^{4}$ Medical Subject Headings $(\mathrm{Mes} H)$, combinando todas ellas mediante el uso de los conectores AND y OR para ganar en amplitud de posibles fuentes secundarias. Los términos utilizados a partir de la consulta en el MesH fueron: Access to health, Geographical Access Barriers, Rural Areas Health. Los filtros o límites aplicados fueron: idiomas (inglés, español y portugués), disponibilidad para descarga en texto completo y periodo de 1990 en adelante. Posteriormente se leyeron los artículos y una síntesis de su contenido se almacenó en un archivo de Excel.

Para la etapa del inventario del talento humano y de prestadores de salud se usaron fuentes secundarias, tales como informes de salud y la base de datos del Ministerio de Salud y Protección Social de Colombia, "Registro Especial de Prestadores de Salud" (REPS), en el cual se documentan los prestadores de servicios de salud general o especialidad, la ubicación y la clasificación según la naturaleza jurídica (pública o privada) y el nivel de atención; se consultó también la página web de gobernación del Tolima y los reportes e informes técnicos registrados formalmente en los distintos micrositios web de esta página.

2. Recolección de datos cuantitativos: el inventario de servicios y talento humano en salud, así como la infraestructura vial, se llevó a cabo mediante acceso a información en línea de la página oficial del Ministerio de Protección Social y Salud del Departamento Administrativo Nacional de Estadística (DANE) y del Instituto Nacional de Vías (INVIAS). En la segunda etapa de la investigación se recolectaron los datos del departamento del Tolima con base en el último reporte disponible en su página, con proyecciones en la demografía al año 2018; también se documentaron en otro archivo de Excel las distancias en kilómetros de cada entidad territorial hasta la capital, Ibagué.

Como segundo paso de esta etapa, se consultó el número de prestadores de salud y salud oral en el departamento desde febrero hasta mayo de 2018, de la base de datos ya mencionada. Al interior de la base se hizo una búsqueda en el subsitio del departamento de Tolima; luego se documentó el número de prestadores de salud: públicos y privados; se clasificaron por nivel de atención, o por complejidad; se enfatizó en los prestadores del servicio de odontología general y de las cinco especialidades odontológicas que el gobierno colombiano ofrece a la población en el sector público.

Se solicitó y obtuvo el mapa del departamento al Instituto Geográfico Agustín Codazzi, entidad del orden nacional avalada para documentar y almacenar los mapas actualizados de cada departamento y región del país, en el que se pueden visualizar las vías de comunicación del departamento, el estado (pavimentado o afirmado, es decir, vías que han sido niveladas y reforzadas con material de piedra usando aplanadoras, sin colocación de asfalto) y características geográficas (Figura 1). Sobre este mapa se midió la distancia de cada uno de los 47 municipios hacia la capital del departamento, usando la escala registrada; cuántos kilómetros de las carreteras están pavimentados y cuántos solamente cuentan con afirmado (rocas trituradas acomodadas mediante aplanadoras). Se trianguló esta

${ }^{4}$ Los tesauros son listados de términos utilizados para representar conceptos. Emplearlos evita problemas relacionados con el uso de varios sinónimos para un mismo vocablo. 
información con la herramienta de Google Maps, calculando los kilómetros entre un sitio y otro, así como el estado de las carreteras informado en la aplicación, característica que puede no coincidir con la realidad (información obtenida en una investigación anterior; Caicedo-Rosero \& Estrada-Montoya, 2016), por lo que en este trabajo se complementó la medición sobre el mapa con mediciones reales en las cuatro salidas de campo.

3. Etapa cualitativa y confirmación de la cuantitativa. Se realizaron cuatro salidas de campo a los municipios de Icononzo, Herveo, Roncesvalles y Planadas, en los cuales se hizo observación no participante, así como entrevistas a algunos/as habitantes, entre ellos/as, personal auxiliar de enfermería y odontólogos/as generales que brindaban atención en los hospitales de primer nivel de las zonas y a personas usuarias de los servicios de salud.

4. Acercamiento interdisciplinario. Mediante el programa AutoCAD se dibujaron distintos mapas que incorporaron los resultados de la investigación previamente almacenados en los diferentes archivos de Excel, con un esqueleto del mapa en el que se corroboraron e incorporaron con las herramientas del programa diferentes colores para diferenciar la distribución de la población, de los prestadores de servicios por niveles y complejidad, así como si se presentaba la barrera de acceso geográfico o no. La totalidad de mapas se almacenó en un Drive de la investigación y está disponible por requerimiento con el autor de correspondencia.

5. Aplicación del sistema de medida de tiempo de viaje para establecer barreras de acceso geográfico en el Tolima. Se aplicó el sistema de medida definido en la investigación "Inventario de talento humano y servicios en salud oral y barreras de acceso geográfico en el departamento de Nariño", en el que se contemplan la distancia y el tiempo; ahí se identificó la duración del viaje que toma el desplazamiento de un municipio de Nariño hasta Pasto en cuatro medios de transporte: a pie, bicicleta, a caballo y vehículo (CaicedoRosero \& Estrada-Montoya, 2016). Se utilizaron los dos criterios de barrera de acceso geográfico avalados por la OMS, así: o un tiempo de viaje mayor a cuatro horas o una distancia mayor a $25 \mathrm{~km}$ desde cada municipio hasta la capital del departamento, ya que allí se ofrecen los niveles más complejos de atención en salud (Ilamados en nuestro país de tercer y cuarto nivel). El método se validó en Colombia identificando el tiempo que tarda una persona en recorrer una distancia calculando la velocidad promedio en un lapso de una hora, tomada en $\mathrm{km} / \mathrm{h}$. Se encontró que el promedio fue: caminando, 5 $\mathrm{km} / \mathrm{h}$; en caballo, $6 \mathrm{~km} / \mathrm{h}$, en bicicleta, $10 \mathrm{~km} / \mathrm{h}$ y en transporte público, $55 \mathrm{~km} / \mathrm{h}$. Este mismo modelo se aplicó en Boyacá y de nuevo en este trabajo, para una vez implementadas las validaciones y ajustes obtenidos en las salidas de campo, conseguir una fórmula más precisa y así después estimar los tiempos de viaje en todo el departamento y en estudios futuros.

Los municipios se seleccionaron basándose en la distancia que tienen respecto de lbagué, tratando de tomar aquellos más alejados en los cuatro puntos cardinales, o con características de dificultad en el acceso, como Planadas, el más distante de todo el departamento; Herveo y Roncesvalles, situados hacia la zona montañosa del Tolima, e Icononzo, debido a que es donde está localizada una zona de reintegración y capacitación para desmovilizados/as de las Fuerzas Armadas Revolucionarias de Colombia (FARC), territorio urbano y rural donde la Universidad Nacional de Colombia implementa proyectos de extensión solidaria. 
Los diferentes viajes se efectuaron en el primer bus hacia Ibagué (los horarios variaron desde las 2 a. m. para Planadas, hasta las 6 a. m. para los otros tres); se optó (en caso de que hubiese más de una opción) por el bus de servicio público más económico (que se conoce en nuestro lenguaje cotidiano como el servicio ordinario) y el registro de la distancia y velocidad promedio de viaje se establecieron con la ayuda de la red social Strava, basada en internet y GPS enfocada en deportistas, como ciclistas y corredores. A ésta la gestiona una compañía del mismo nombre con sede en San Francisco y permite conocer la velocidad promedio recorrida por tramos con gran precisión y así fue anotada en el archivo de Excel, para "servicio de transporte público" en carretera pavimentada y afirmada, iniciando la medición desde que se tomaba el bus hasta que llegaba a la terminal de transportes de lbagué.

Con los resultados de la velocidad de $\mathrm{km} / \mathrm{h}$ en pavimentado $(52 \mathrm{~km} / \mathrm{h})$ y afirmado $(17 \mathrm{~km} / \mathrm{h})$ de la etapa anterior, en un archivo de Excel se hizo el cálculo promedio para la totalidad de los municipios (incluidos los cuatro de la salida de campo). En el mapa de carreteras, siguiendo las convenciones allí establecidas, se dividió la distancia total de kilómetros en tramos pavimentados y afirmados, y se computó el tiempo de viaje combinando las dos velocidades de 52 y $17 \mathrm{~km} / \mathrm{h}$.

6. Consolidación y análisis de la información en bases de datos. Una vez hechos todos los cálculos, se clasificaron e integraron los datos en otro archivo de Excel para tener una visión de conjunto de toda la investigación: inventario de prestadores de salud y salud oral en el departamento, demografía por sexo, prestadores de salud públicos y privados, prestadores de salud en odontología general y especializada pública y privada, medidas de tiempo y distancia a Ibagué, y cálculo de la proporción odontólogo/a-paciente por municipio. Esto último se determinó con el análisis de la relación de un/a odontólogo/a por cada 2700 habitantes propuesta por la OMS como ideal en cada municipio y clasificando los resultados en aquellos que cumplían con el promedio, así como los que tenían necesidad de uno, dos, tres, cuatro a 10 y más de 10 odontólogos/as.

\section{Resultados}

\section{Organización territorial, estructura y distribución de los prestadores de servicios de salud}

El departamento del Tolima se encuentra ubicado en el centro-oeste del país, con una extensión de 23562 km² (Gobernación del Tolima, 2018). La división territorial del departamento está conformada por 47 municipios y su capital es Ibagué. Cuenta con una población proyectada para el 2018 de 1419947 habitantes, de los cuales $69.07 \%$ se encuentran en la cabecera y el resto es población dispersa. Se caracteriza por tener todos los pisos térmicos (cálido, templado, frío, páramo y nevados); la producción económica, en gran parte, se establece por el sector primario y el terciario, siendo el comercio (transporte, almacenamiento y comunicaciones) una de las principales actividades de la región, junto con la agricultura, ganadería, caza, silvicultura y pesca (Gobernación del Tolima, 2018).

Los datos de la demografía y los niveles de atención del departamento se recogen en el Cuadro 1 , que contiene la información disponible más reciente. Todos los municipios del departamento del Tolima cuentan con hospitales públicos de primer nivel; del segundo nivel hay en Chaparral, Espinal, Honda, Líbano, Purificación y Lérida; del tercer nivel de atención se encuentra solamente el hospital Federico Lleras Acosta, Empresa Social del Estado (ESE), en Ibagué, y debe atender a todos los habitantes del Tolima que requieran alguno de sus servicios. De esta situación, común en casi 
todos los departamentos colombianos, se colige que si alguna persona del departamento necesita servicios hospitalarios y especializados, debe desplazarse hasta la ciudad de Ibagué, lo que para los lugares más alejados se traduce -de entrada- en una barrera de acceso geográfico, pues implica el traslado del o de la paciente con o sin acompañante, muchas veces desde el día anterior a la cita programada y hasta un día después, lo que representa un costo mayor no solo en términos económicos, sino en el de los itinerarios a los que se ven sometidos y el aumento del sufrimiento para muchos/ as de los/as habitantes de los municipios ubicados en las periferias. ${ }^{5}$

El dato de los prestadores de salud en el sector privado para el departamento del Tolima también se encontró en el registro de los REPS de la página web oficial del Ministerio de Salud y Protección Social. Dicho sector se clasifica por complejidad en: baja, media y alta, para diferenciarla del sector público, pero para efectos de la interpretación en esta investigación se pueden considerar equivalentes. Los resultados arrojaron un total de prestadores de baja complejidad de 1 643, con más de $50 \%$ de ellos concentrados/as en la ciudad de Ibagué. De los 47 municipios, solo 10, además de la capital, cuentan con la presencia del sector privado, dejando al restante $76.6 \%$ de los municipios solamente con los servicios públicos.

\section{Recursos humanos en salud oral}

La información consignada en este inventario se obtuvo consultando la página oficial del Ministerio de Salud y Protección Social, en "Registro Actual", y se utilizó el filtro por departamento, municipio, servicio de odontología general y todos/as los/as diferentes especialistas en el sector público y privado, incluyendo instituciones prestadoras de salud y profesionales independientes.
El primer nivel de atención comprende servicio de odontología general, que se encarga de los programas de promoción y prevención de forma obligatoria en todos/as los/as pacientes y se debe brindar en el momento en que el/la paciente inicie su tratamiento odontológico. Puesto que todos los municipios cuentan con el primer nivel de atención, esto indicaría universalidad en cuanto al servicio de odontología general, pero no significa que los servicios prestados sean suficientes para suplir las necesidades de la población. En total existen 101 registros de prestadores de servicios de odontología general en el departamento, con un mayor porcentaje en la ciudad de Ibagué (Cuadro 2).

En el sector privado la odontología general se ejerce desde un nivel de atención de baja complejidad, pero se determina de acuerdo con la relación de oferta y demanda, dejando de lado las obligaciones que sí tiene el sector público, como son la promoción y la prevención. En cuanto a la distribución, es evidente una concentración en la ciudad de Ibagué, con $63.44 \%$.

La elaboración del inventario en relación con la odontología especializada se hizo consultando las bases de datos del Ministerio de Salud y Protección Social, en la sección "Registro Actual", las cuales nos permitieron encontrar el número total de especialistas en odontología a nivel público y privado en el Tolima, que fue de 263 especialistas, con 100 de ellos en el campo de la ortodoncia, 68 ubicados en la ciudad de Ibagué. Un $80.60 \%$ de especialistas de todos los campos están concentrados en la capital, lo que se configura en una barrera de acceso, pues significa un mayor tiempo de viaje y de costos con la finalidad de acceder a estos servicios para los/as habitantes de los municipios del departamento. En

${ }^{5}$ En uno de los viajes, la investigadora pudo constatar la experiencia de sufrimiento de la separación de un núcleo familiar (del régimen subsidiado), toda vez que el padre trabaja una hora más al sur de Planadas y su esposa y bebé recién nacido requieren citas con especialista en labio y paladar hendido que solo se otorgan en Ibagué, para lo cual la madre y el bebé viajan hasta esa ciudad, se hospedan con amigas/os o familiares lejanos y él, cuando puede, toma días libres de su trabajo como jornalero para ir a visitarlos. Al ser trabajador por cuenta propia, los días no laborados se traducen en jornadas sin ingreso y gastos de desplazamiento hasta Ibagué que se suman a los de su familia. 
el sector público encontramos las especialidades de cirugía maxilofacial, cirugía oral, endodoncia y ortodoncia, con un total de siete especialistas para todo el departamento, cuatro localizados en Honda, con todas las especialidades mencionadas, dos en Ibagué y uno en Espinal.

La proporción odontólogo/a-paciente fue establecida para odontólogos/as generales del sector público con la información registrada previamente. Se efectuó este cálculo usando una regla de tres simple para conocer la relación ideal de odontólogos/as según la cantidad de habitantes de cada municipio. A la relación ideal se le restó el número de especialistas en cada municipio para obtener un valor, que representa el número de odontólogos/as faltantes para cumplir con ésta. Establecido el número de faltantes, se organizaron los municipios de acuerdo con el requerimiento adicional de odontólogos/as en: uno más, dos más, tres más, de cuatro a 10, y mayor a 10, y también los que cumplían con la relación ideal. Es evidente el requerimiento de odontólogos/ as en la mayoría de municipios desde el sector público, empezando por la capital del departamento y los municipios con más habitantes. Solamente en tres existe la relación ideal, lo que demuestra la falta de fortalecimiento del sector público en salud oral y todas las obligaciones que tiene implica que no están reguladas desde el sector privado, como el importante papel de los programas de promoción y prevención (Cuadro 3).

\section{Determinación de las barreras de acceso geográfico a salud según distancia y tiempo}

En esta parte del estudio se consultó la distancia de los 47 municipios del departamento hasta la capital, Ibagué, donde existen todos los niveles de atención en sector público y privado, con profesionales en todas las áreas de la salud y de salud oral. Con los datos de la distancia de los municipios hasta Ibagué se aplicó el modelo para identificar el tiempo de viaje que le tomaría a alguien desplazarse hasta la capital en los diferentes medios de transporte elegidos: caminando, bicicleta, caballo y automóvil.

Todos los municipios presentan barreras de acceso geográfico a servicios de salud en toda la extensión del departamento y la gente requiere un tiempo mayor a cuatro horas para trasladarse caminando de su lugar de residencia hasta una institución de salud del tercer nivel. En el desplazamiento en bicicleta se observa que más de $90 \%$ de los municipios presentan barrera de acceso geográfico y solamente tres no tienen esta característica por estar más cerca de la ciudad, aunque se hallan por encima de las tres horas de viaje. En el recorrido en automóvil encontramos que hay barrera de acceso geográfico a servicios de salud solamente en el municipio de Planadas, ubicado al sur del departamento.

Esta investigación tuvo el interés particular de llevar a cabo un trabajo de campo en cuatro de los municipios del departamento. Se calculó la velocidad promedio asumiendo una velocidad constante y estado igual de la carretera durante todo el trayecto. Tres de los municipios, Roncesvalles, Herveo y Planadas, presentan la barrera de acceso relacionada con el tiempo de viaje y la distancia en km (Cuadro 4).

Los resultados de la aplicación del modelo ajustado a todos los municipios del departamento, bajo la presunción de que las condiciones topográficas desde y durante los viajes son similares, los modificó levemente respecto de las investigaciones anteriores, arriba mencionadas. Esta presunción, como se pudo confirmar en los cuatro municipios, no es homogénea, dado el estado diferencial de las carreteras. Según se aprecia en el Cuadro 5, los municipios de Herveo, Planadas, Rioblanco, Roncesvalles, San Antonio y Villa Hermosa presentan barrera de acceso a los servicios de salud de la ciudad de Ibagué cuando se aplica el modelo ajustado, si se mantuvieran las mismas condiciones en el estado de las carreteras.

En los desplazamientos a cuatro municipios se realizó observación etnográfica y entrevistas en los hospitales de primer nivel acerca de los servicios 
que se prestan en odontología, a qué hospitales se hacen las remisiones $y$, a partir de esto, se recopiló la información de interés para esta investigación. También se tuvieron en cuenta las experiencias de la investigadora en el recorrido hasta Ibagué desde cada municipio.

\section{Aproximación cualitativa}

En Icononzo se visitó el Hospital Sumapaz ESE, que presta los servicios de urgencias, consulta externa por medicina y odontología general. Dependiendo de los convenios que tienen los/as pacientes con las EPS dentro de la red complementaria, las remisiones se realizan a Girardot o Ibagué, considerando la complejidad de la atención que se necesita y la disponibilidad de camas en el centro asistencial; hay ocasiones en que los traslados se hacen al Líbano o a Neiva, ubicados en otro departamento, en alguna de las tres ambulancias que tiene el hospital. La mayoría de los/as habitantes cuenta con aseguramiento subsidiado, mayormente afiliados/as a la Nueva Empresa Pomotora de Salud del sector público (conocida como nueva EPS).

La odontóloga encargada de la atención informa que brinda los servicios reglamentados de promoción y prevención, operatoria, raspaje supragingival y exodoncias; reporta no realizar, hasta el momento, alguna endodoncia (desde diciembre de 2017). Estos procedimeintos se efectúan solamente en dientes anteriores, debido a que el hospital no cuenta con servicio de radiología intraoral, por lo que, en caso de requerirse, el centro radiológico más cercano está en Melgar, a una hora de distancia. La odontóloga encargada no suele indicar remisiones debido al tiempo que implican las autorizaciones, pero en caso de hacerlas, suelen ser hacia Ibagué, Espinal o Girardot. Cuando se presentan urgencias por dolor extremo, normalmente practica una exodoncia. La población en la zona de reintegración y capacitación de desmovilizados de las FARC, ubicada en la vereda La fila, cuenta con un médico para las personas que posean un tipo de identificación llamado código (mientras completan el proceso de identificación con cédula de ciudadanía), que les permite acceder a una EPS. Este trámite ha sido llevado a cabo por casi todos los desmovilizados/as con la nueva EPS.

En el recorrido por el municipio se identificaron tres centros odontológicos. Uno ofrece los servicios de ortodoncia, endodoncia, rehabilitación oral y cirugía maxilofacial, lo que contrasta con el registro de prestadores que informa la presencia de dos odontólogos/as generales y ninguna especialidad. En conversación con una habitante del lugar, dedicada a la venta ambulante, se pudo conocer que suele ir a tratamientos odontológicos de rehabilitación en los consultorios privados del municipio y para las urgencias, cuando no son a altas horas de la noche, en el hospital. El viaje de regreso por bus a Ibagué inició a las 4:00 a. m. El transporte hace una parada de media hora en la ciudad de Melgar. Los buses, que se dirigen de Icononzo a Melgar, salen aproximadamente cada dos horas desde las 4:00 a. m. hasta las 10:00 p. m. La carretera, durante los primeros 30 minutos de marcha, presenta tramos que no están en óptimas condiciones, aunque está pavimentada.

En el hospital en Planadas las remisiones dependen de los convenios que tiene la EPS con los nosocomios y los lugares más habituales a los que se realizan son a Chaparral y al Espinal, que cuentan con segundo nivel de atención, o a Ibagué, con tercer nivel. El odontólogo encargado refirió brindar los servicios reglamentados de promoción y prevención, operatoria, raspaje supragingival y exodoncias, así como terapias endodónticas completas en dientes unirradiculares y birradiculares; ofrece remisiones a endodoncia y a cirugía maxilofacial dependiendo de la necesidad del o de la paciente. El hospital cuenta con radiología intraoral. Las urgencias que se pueden atender consisten en pulpotomías más que exodoncias, para calmar el dolor. En cuanto a los/as odontólogos/as de práctica privada, según el registro de prestadores de servicios de salud en el municipio, hay 
tres en el hospital, tres en consultorios privados y un ortodoncista. El odontólogo comenta sobre la dificultad en el transporte, debido a los diferentes derrumbes que se ocasionan en épocas de lluvias.

La entrevista con una habitante del municipio que trabaja en el rubro de hospedaje permitió identificar algunas características sobre el tiempo y condiciones del desplazamiento para asistir a consultas médicas en Ibagué. Ella comentó que el transporte depende del estado de los buses y de las condiciones de la carretera, que suelen empeorar en la temporada de lluvias. Ello hace que el tiempo de recorrido sea de entre cinco y ocho horas, lo que obliga a salir con un día de anticipación si la cita está programada para las primeras horas de la mañana y a quedarse a dormir en Ibagué, ciudad en la cual unos familiares residen, para madrugar y poder acudir a tiempo. Lo anterior le implica disponer de más de dos días para cada consulta. Acerca de la atención odontológica, comenta que utiliza los servicios privados. A las 2:00 a. m. se abordó el bus con destino a Ibagué. Toda la vía, hasta Ataco, está totalmente afirmada. Oficialmente, el viaje tiene una duración de siete horas. El bus se encontró en malas condiciones técnicas, con un tiempo de viaje de alrededor de las siete horas, lo que comprueba las dificultades y barreras a las que se enfrentan los/ as habitantes de este municipio, el más alejado del departamento.

En el hospital San Antonio, en Herveo, las remisiones y traslados se hacen hacia Honda, Manizales (ubicada en un departamento vecino), Líbano e Ibagué. Ibagué y Manizales cuentan cuentan servicios de tercer nivel y en Líbano hay servicios de alta complejidad en cuidados intensivos. Estos trámites dependen del convenio que tenga la EPS y la disponibilidad de camas en los sitios a donde son remitidos, información que se obtiene por teléfono.

En cuanto a odontología, la profesional encargada refirió cumplir los mismos procedimientos simples. Nunca ha hecho alguna remisión, debido al tiempo que toman las autorizaciones para los/as afiliados/as a régimen subsidiado, por lo que recurre, en su mayoría, a exodoncias. Este hospital no cuenta con radiología intraoral, de ahí que no se practican endodoncias y las pulpotomías se hacen calculando la longitud del diente. También comenta que en la atención privada hay un odontólogo especialista de la ciudad de Manizales que se presenta una vez al mes para supervisar tratamientos de endodoncia y rehabilitación oral, pero que también conoce a otra persona que se ocupa de tratamientos paliativos e inclusive coloca aparatología ortodóntica fija a pacientes pediátricos/as, por lo que muchas veces ella tiene que atender urgencias por procedimientos hechos fuera de su consulta, mismos que suele negar para evitar problemas legales. Manifiesta haber presentado quejas acerca de las prácticas de esta persona empírica, pero no ha tenido resultados con las denuncias. En el registro del Ministerio de Salud y Protección Social aparece la prestadora del sector público de este hospital de primer nivel.

El viaje a Roncesvalles se culminó en horas de la noche, debido a los derrumbes en la carretera al momento del viaje; las entrevistas se realizaron el día domingo. El hospital Santa Lucía (ESE) tiene infraestructura en buen estado y cuenta con una adecuada organización y atención de sus trabajadores/as. La EPS se encarga directamente de las remisiones y ubicación de los/as pacientes que, en su mayoría, están en régimen subsidiado: nueva EPS. A la odontóloga que en ese momento cursaba su año de servicio social obligatorio (conocido en Colombia como año de práctica rural o simplemente, rural) no se le pudo contactar, porque trabaja de martes a sábado y los lunes hace brigadas en las veredas. En la cabecera municipal se identificó un laboratorio donde se confeccionan prótesis sin la presencia del servicio de odontología. El único prestador encontrado en la base de datos REPS del Ministerio de Salud y Protección Social es el del hospital.

Se tomó el bus de regreso a Ibagué a las 10:00 a. m. Al salir de la cabecera municipal, en la 
carretera afirmada hubo varios tramos con derrumbes e inconvenientes para seguir el camino por los deslizamientos que ocurren intermitentemente, hasta el municipio de Rovira. Si bien existen vías que comunican en menor distancia a distintos municipios, su estado solo permite el tránsito de vehículos particulares con tracción en las cuatro ruedas. Los trayectos se hacen por las rutas que están en mejores condiciones, a pesar de que impliquen mayor tiempo o distancia, e inclusive que sirvan a los intereses económicos de los transportadores privados quienes, en una sola ruta, abarcan varios municipios entre las microrregiones o en el interior de ellas.

\section{Discusión y conclusiones}

En la indagación se encontraron varios aspectos positivos en cuanto a la diversidad de climas, culturas y producción, lo que hace al departamento del Tolima abundante en elementos que también representan al país y contienen muchos de los atributos que vuelven a Colombia un país inmensamente rico. La disposición geográfica del departamento, encontrándose en una zona central, con el paso por su territorio de las cordilleras Central y Oriental, de Los Andes, del Río Magdalena que lo atraviesa, y la infraestructura de comunicación terrestre como es la vía que va desde Ibagué hasta Armenia, que permite el trasporte de personas y mercancías hacia las zonas del occidente y sur-occidente del país, lo colocan en un lugar privilegiado en el mapa y se esperaría que la infraestructura vial se correspondiera con este primerísimo sitio en el plano comercial.

En el ámbito del talento humano en salud oral, este estudio sugiere que es necesario fortalecerlo para que al menos en la relación cuantitativa se garantice la cobertura a todos/as los/as habitantes del departamento e implementar los distintos programas de la gobernación con profesionales suficientes, sobre todo en lugares como la capital, Chaparral, Espinal, Guamo, Líbano, Mariquita y Melgar, con un déficit de más de 10 odontólogos/as. Aunque tiene una fuerte presencia el sector privado en esta especialidad, manifiesta una desigualdad en cuanto al derecho a acceder a la salud oral, ya que hay un contrato de prestación privada de servicios de por medio, regido por leyes del mercado.

Basados en el trabajo empírico llevado a cabo en este proyecto, es importante recalcar las barreras que deben superar las personas de los distintos municipios, en general el importante papel que juegan las EPS y las afiliaciones que éstas tengan con la red complementaria en salud, la disponibilidad de las camas para recibir al o a la paciente, lo que nos habla de una carencia en el cupo de atención, tema que puede tratarse en otra investigación. Son cuestionables las características del sistema de aseguramiento cuando la desigualdad es evidente en la centralización de servicios de alta complejidad, la inequidad en salud basada en el régimen contributivo al que se está inscrito/a, que permite elegir una EPS de mayor calidad. En el caso de Planadas, Herveo y Roncesvalles, están relacionados directamente con el estado de las carreteras pavimentadas y afirmadas, con la calidad en el servicio de transporte público, paradas e intermitencia en los horarios de llegada y salida de los buses a cada uno de los municipios, desde y hacia Ibagué.

Al inicio de la investigación se esperaba que la atención en salud fuera prestada en municipios o ciudades pertenecientes a otro departamento para los municipios ubicados en sus fronteras, situación que en el caso de Herveo -con la atención en la ciudad de Manizales- y para Icononzo -con el municipio de Girardot, en un departamento vecinoquedó documentada. Análisis como este, realizado en el departamento del Tolima, al sur de la capital del país, nos permiten como universidad pública lograr avances importantes de tipo empírico para seguir profundizando en la evidencia de las diferentes barreras de acceso a los servicios de salud y salud oral a lo largo y ancho de nuestro país, más allá de los obstáculos geográficos y los de la proporción 
odontólogo/a-paciente, pues empiezan a emerger impedimentos de tipo institucional, cultural y los concernientes a las múltiples barreras del sistema de salud, denominadas por Abadia \& Oviedo (2009) como itinerarios burocráticos en salud.

\section{Referencias}

Abadia, C. E. \& Oviedo, D. G. (2009). Bureaucratic Itineraries in Colombia. A theoretical and methodological tool to assess managed-care health care systems. Social Science \& Medicine, 68, 11531160.

Beyer, K. M., Saftlas, A. F., Wallis, A. B., Peek-Asa, C. \& Rushton, G. (2011). A probabilistic sampling method (PSM) forestimating geographic distance to health services when only the region of residence is known. International journal of health geographics, 10, 4. https://doi.org/10.1186/1476-072X-10-4

Braveman, P. S. (2003). Defining equity in health. Journal of Epidemiol Community Health, 57, 254258.

Caicedo-Rosero, A. V. \& Estrada-Montoya, J. H. (2016). Barreras geográficas de acceso a los servicios de salud oral en el departamento de Nariño, Colombia. Revista Gerencia y Políticas en Salud, 15(31), 146-174.

Comber, B. C. \& Radbum, R. (2011). A spatial analysis of variation in health access: linking geography, socio - economic status and access perception. International Journal of Health Geographics, 10-44.

Congreso de la República. (1993). Ley 100 de 1993; Por la cual se crea el sistema de seguridad social integral y se dictan otras disposiciones. Bogotá: Congreso de la República.

Estrada-Montoya, J. H. \& Pulido, D. (2012). Barreras de acceso geográficas en el departamento de Boyacá (Trabajo de grado presentado para optar por el título de odontóloga), Universidad Nacional de Colombia.
Gao, S., Mioc, D., Anton, F., Yi, X. \& Coleman, D. J. (2008). Online GIS services for mapping and sharing disease information. International Journal of health Geographics, 7. https://doi.org/10.1186/1476-072X-7-8

Gobernación del Tolima. (2018). Página web oficial de la gobernación. Consultado en http://www.tolima.gov.co/.

Guagliardo, M. F. (2004). Spatial accessibility of primary care: concepts, methods and challenges. International Journal of health Geographics, 3, 1-13. https://doi.org/10.1186/1476-072X-3-3

Gulliford, M. M. (2003). Access to health care. London: Routledge.

Jordan, R. P., Martín, D. \& Barnett, S. (2004). Distance, rurality and the need for care: access to health services in South West England. International Journal of Health Geographics, 3.

Kirby,J.B.\&Kaneda, T.(2005). Neighborhood Socioeconomic Disadvantage and Access to Health Care. Journal of Health and Social Behavior, 46, 15-31. https://doi.org/10.1177/002214650504600103

Magilvy, J. C. (2000). The crisis nature of health care transition for rural older adults. Public Health Nurs, 17(5), 336-345.

Mauch, W. N., Kirubi, K. H., Sitienei, J. \& Klinkenberg, E. (2011). Assessing access barriers to tuberculosis care with the tool to estimate patients'cost: pilot results from two districts in Kenya. BMC Public Health, 11(43), 1-9.

Ministerio de Salud y Protección Social. Sede Web del Ministerio Salud y Protección Social. Colombia, 2018. Recuperado de http://www.minsalud.gov.co/ salud/Paginas/INDICADORESBASICOSSP.aspx.

Nuckols, J. W. \& Jarup, L. (2004). Using Geographic information systems for exposure assessment in environmental epidemiology studies. Enviromental health perspectives, 112(9), 1007-1015. 
Qidwai, W., Ashfaq, T., Khoja, T. A., Rawaf, S., Kurashi, N. Y. \& Alnasir, F. (2012). Access to person-centered care: a perspective on status, barriers, opportunities and challenges from the Eastern Mediterranean Region. Middle East Journal of Family Medicine, 10(6), 4-13.

Von Elm, E., Altman, D. G., Eggera, M., Pocock, S. J., Gotzsche, P. C. \& Vandenbroucke, J. P. (2008). Declaración de la Iniciativa STROBE (Strengthening the Reporting of Observational studies in Epidemiology): directrices para la comunicación de estudios observacionales. Gaceta Sanitaria, 22(2), 144-150.
World Health Organization. (2010). Monitoring equity in access to AIDS treatment programmes: a review of concepts, models, methods and indicators. Francia: autor.

World Health Organization. (2016). Global strategy on human resources for health: workforce 2030. Ginebra: autor. Recuperado de https://www.who.int/ hrh/resources/pub_globstrathrh-2030/en/

Zhang, D. M., Qiu, L., Zhou, W., Zhao, Y. \& Gu, D. (2017). Urban-rural differences in the association between access to healthcare and health outcomes among older adults in China. BMC Geriatrics, 17(1), 151. 


\section{Figura 1.}

Mapa del departamento del Tolima

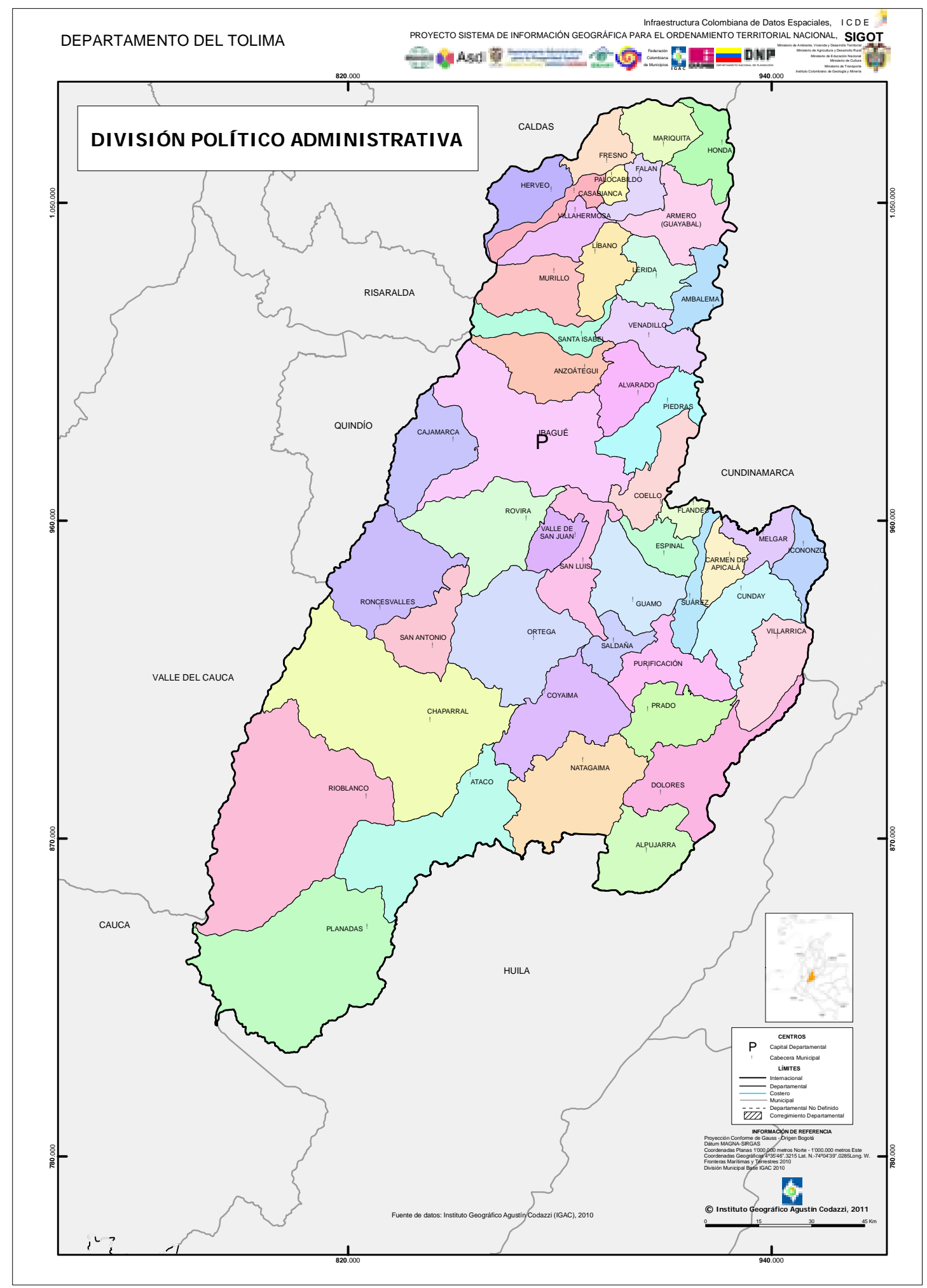

Fuente: Instituto Geográfico Agustín Codazzi. 
Cuadro 1.

Demografía del departamento del Tolima y distribución de los niveles de atención pública y privada por municipio

\begin{tabular}{|c|c|c|c|c|c|c|c|c|c|c|c|c|}
\hline \multirow{3}{*}{ Municipio } & \multicolumn{3}{|c|}{ Demografía } & \multicolumn{9}{|c|}{ Nivel de atención } \\
\hline & \multirow[t]{2}{*}{ Hombres } & \multirow[t]{2}{*}{ Mujeres } & \multirow[t]{2}{*}{ Total } & \multicolumn{4}{|c|}{ Público } & \multicolumn{4}{|c|}{ Privado } & \multirow[t]{2}{*}{ Total } \\
\hline & & & & Primero & Segundo & Tercero & Subtotal & Bajac & Mediac & Attac & Subtotal & \\
\hline Ibagué & 271,360 & 287,445 & 558,805 & 37 & 0 & 1 & 38 & 1035 & 1203 & 113 & 2351 & 2389 \\
\hline Alpujarra & 2,598 & 2,376 & 4,974 & 2 & 0 & 0 & 2 & 0 & 0 & 0 & 0 & 2 \\
\hline Alvarado & 4,483 & 4,333 & 8,816 & 1 & 0 & 0 & 1 & 0 & 0 & 0 & 0 & 1 \\
\hline Ambalema & 3,480 & 3,275 & 6,755 & 1 & 0 & 0 & 1 & 0 & 0 & 0 & 0 & 1 \\
\hline Anzoátegui & 10,122 & 8,516 & 18,638 & 1 & 0 & 0 & 1 & 0 & 0 & 0 & 0 & 1 \\
\hline Armero - Guayabal & 6,038 & 5,801 & 11,839 & 1 & 0 & 0 & 1 & 1 & 0 & 0 & 1 & 2 \\
\hline Ataco & 11,312 & 11,277 & 22,589 & 2 & 0 & 0 & 2 & 0 & 0 & 0 & 0 & 2 \\
\hline Cajamarca & 10,263 & 9,378 & 19,641 & 2 & 0 & 0 & 2 & 20 & 0 & 0 & 20 & 22 \\
\hline Carmen de Apicalá & 4,454 & 4,381 & 8,835 & 1 & 0 & 0 & 1 & 0 & 0 & 0 & 0 & 1 \\
\hline Casabianca & 3,591 & 3,070 & 6,661 & 2 & 0 & 0 & 2 & 0 & 0 & 0 & 0 & 2 \\
\hline Chaparral & 23,615 & 23,633 & 47,248 & 2 & 1 & 0 & 3 & 44 & 6 & 1 & 51 & 54 \\
\hline Coello & 5,172 & 4,638 & 9,810 & 1 & 0 & 0 & 1 & 0 & 0 & 0 & 0 & 1 \\
\hline Coyaima & 14,857 & 13,478 & 28,335 & 3 & 0 & 0 & 3 & 5 & 0 & 0 & 5 & 8 \\
\hline Cunday & 5,042 & 4,592 & 9,634 & 3 & 0 & 0 & 3 & 0 & 0 & 0 & 0 & 3 \\
\hline Dolores & 4,186 & 3,829 & 8,015 & 1 & 0 & 0 & 1 & 0 & 0 & 0 & 0 & 1 \\
\hline El Espinal & 38,228 & 37,921 & 76,149 & 1 & 1 & 0 & 2 & 158 & 63 & 5 & 226 & 228 \\
\hline Falan & 4,650 & 4,561 & 9,211 & 1 & 0 & 0 & 1 & 0 & 0 & 0 & 0 & 1 \\
\hline Flandes & 14,416 & 14,783 & 29,199 & 1 & 0 & 0 & 1 & 2 & 0 & 0 & 2 & 3 \\
\hline Fresno & 15,600 & 14,565 & 30,165 & 1 & 0 & 0 & 1 & 24 & 1 & 0 & 25 & 26 \\
\hline El Guamo & 15,754 & 16,359 & 32,113 & 1 & 0 & 0 & 1 & 60 & 13 & 0 & 73 & 74 \\
\hline Herveo & 4,203 & 3,805 & 8,008 & 1 & 0 & 0 & 1 & 0 & 0 & 0 & 0 & 1 \\
\hline Honda & 12,867 & 11,680 & 24,547 & 0 & 1 & 0 & 1 & 70 & 22 & 6 & 98 & 99 \\
\hline Icononzo & 5,527 & 5,367 & 10,894 & 1 & 0 & 0 & 1 & 0 & 0 & 0 & 0 & 1 \\
\hline Lérida & 8,468 & 8,927 & 17,395 & 0 & 2 & 0 & 2 & 22 & 1 & 0 & 23 & 25 \\
\hline Líbano & 20,285 & 19,981 & 40,266 & 0 & 1 & 0 & 1 & 53 & 14 & 3 & 70 & 71 \\
\hline Mariquita & 16,502 & 16,827 & 33,329 & 1 & 0 & 0 & 1 & 63 & 12 & 0 & 75 & 76 \\
\hline Melgar & 18,542 & 17,797 & 36,339 & 1 & 0 & 0 & 1 & 61 & 6 & 0 & 67 & 68 \\
\hline Murillo & 2,695 & 2,323 & 5,018 & 1 & 0 & 0 & 1 & 0 & 0 & 0 & 0 & 1 \\
\hline Natagaima & 11,284 & 11,232 & 22,516 & 2 & 0 & 0 & 2 & 6 & 0 & 0 & 6 & 8 \\
\hline Ortega & 16,666 & 15,765 & 32,431 & 2 & 0 & 0 & 2 & 8 & 0 & 0 & 8 & 10 \\
\hline Palocabildo & 4,737 & 4,423 & 9,160 & 1 & 0 & 0 & 1 & 1 & 0 & 0 & 1 & 2 \\
\hline Piedras & 2,976 & 2,664 & 5,640 & 2 & 0 & 0 & 2 & 0 & 0 & 0 & 0 & 2 \\
\hline Planadas & 15,605 & 14,369 & 29,974 & 3 & 0 & 0 & 3 & 0 & 0 & 0 & 0 & 3 \\
\hline Prado & 3,862 & 3,839 & 7,701 & 1 & 0 & 0 & 1 & 0 & 0 & 0 & 0 & 1 \\
\hline Purificación & 14,741 & 14,671 & 29,412 & 0 & 1 & 0 & 1 & 6 & 2 & 0 & 8 & 9 \\
\hline
\end{tabular}




\begin{tabular}{|c|c|c|c|c|c|c|c|c|c|c|c|c|}
\hline \multirow{3}{*}{ Municipio } & \multicolumn{3}{|c|}{ Demografía } & \multicolumn{9}{|c|}{ Nivel de atención } \\
\hline & \multirow[t]{2}{*}{ Hombres } & \multirow[t]{2}{*}{ Mujeres } & \multirow[t]{2}{*}{ Total } & \multicolumn{4}{|c|}{ Público } & \multicolumn{4}{|c|}{ Privado } & \multirow[t]{2}{*}{ Total } \\
\hline & & & & Primero & Segundo & Tercero & Subtotal & Bajac & Mediac & Altac & Subtotal & \\
\hline Rioblanco & 12,867 & 11,592 & 24,459 & 1 & 0 & 0 & 1 & 0 & 0 & 0 & 0 & 1 \\
\hline Roncesvalles & 3,315 & 3,029 & 6,344 & 1 & 0 & 0 & 1 & 0 & 0 & 0 & 0 & 1 \\
\hline Rovira & 10,558 & 9,984 & 20,542 & 1 & 0 & 0 & 1 & 0 & 0 & 0 & 0 & 1 \\
\hline Saldaña & 7,206 & 7,179 & 14,385 & 1 & 0 & 0 & 1 & 1 & 0 & 0 & 1 & 2 \\
\hline San Antonio & 7,353 & 6,957 & 14,310 & 3 & 0 & 0 & 3 & 1 & 0 & 0 & 1 & 4 \\
\hline San Luis & 9,395 & 9,758 & 19,153 & 2 & 0 & 0 & 2 & 1 & 0 & 0 & 1 & 3 \\
\hline Santa Isabel & 3,414 & 2,943 & 6,357 & 1 & 0 & 0 & 1 & 0 & 0 & 0 & 0 & 1 \\
\hline Suárez & 2,232 & 2,315 & 4,547 & 1 & 0 & 0 & 1 & 0 & 0 & 0 & 0 & 1 \\
\hline Valle de San Juan & 3,393 & 2,975 & 6,368 & 1 & 0 & 0 & 1 & 0 & 0 & 0 & 0 & 1 \\
\hline Venadillo & 9,996 & 9,656 & 19,652 & 1 & 0 & 0 & 1 & 1 & 1 & 0 & 2 & 3 \\
\hline Villahermosa & 5,743 & 4,909 & 10,652 & 1 & 0 & 0 & 1 & 0 & 0 & 0 & 0 & 1 \\
\hline Villarrica & 2,814 & 2,575 & 5,389 & 1 & 0 & 0 & 1 & 0 & 0 & 0 & 0 & 1 \\
\hline Total & 706,467 & 705,753 & $1,412,220$ & 96 & 7 & 1 & 104 & 1643 & 1344 & 128 & 3115 & 3219 \\
\hline
\end{tabular}

Fuente: Elaborado a partir de la información consignada en las páginas electrónicas del Ministerio de la Salud y del Departamento del Tolima. 
Cuadro 2.

Distribución de odontología general en los sectores público y privado

\begin{tabular}{|l|r|r|r|r|}
\hline \multicolumn{4}{|c|}{ ODONTOLOGÍA GENERAL } \\
\hline \multicolumn{1}{|c|}{ Municipio } & Sector público & Sector privado \\
\hline Cantidad & Porcentaje & Cantidad & Porcentaje \\
\hline Ibagué & 38 & $38.70 \%$ & 177 & $63.44 \%$ \\
\hline Alpujarra & 2 & $2.15 \%$ & 0 & $0 \%$ \\
\hline Alvarado & 1 & $1.08 \%$ & 0 & $0 \%$ \\
\hline Ambalema & 1 & $1.08 \%$ & 0 & $0 \%$ \\
\hline Anzoátegui & 1 & $1.08 \%$ & 0 & $0 \%$ \\
\hline Armero- Guayabal & 1 & $1.08 \%$ & 2 & $0.72 \%$ \\
\hline Ataco & 2 & $2.15 \%$ & 0 & $0 \%$ \\
\hline Cajamarca & 2 & $2.15 \%$ & 2 & $0.72 \%$ \\
\hline Carmen de Apicalá & 1 & $1.08 \%$ & 1 & $0.36 \%$ \\
\hline Casabianca & 2 & $2.15 \%$ & 0 & $0 \%$ \\
\hline Chaparral & 2 & $2.15 \%$ & 7 & $2.51 \%$ \\
\hline Coello & 1 & $1.08 \%$ & 0 & $0 \%$ \\
\hline Coyaima & 1 & $1.08 \%$ & 3 & $1.08 \%$ \\
\hline Cunday & 1 & $3.22 \%$ & 2 & $0.72 \%$ \\
\hline Dolores & 1 & $1.08 \%$ & 0 & $0 \%$ \\
\hline El Espinal & 1 & $1.08 \%$ & 0 & $0 \%$ \\
\hline Falan & 1 & $3.22 \%$ & 10 & $5.02 \%$ \\
\hline Flandes & 1 & $1.08 \%$ & 0 & $0 \%$ \\
\hline Fresno & $1.08 \%$ & 0 & $0 \%$ \\
\hline El Guamo & $1.08 \%$ & 8 & $2.87 \%$ \\
\hline Herveo & $1.08 \%$ & 0 & $3.23 \%$ \\
\hline Honda & $1.08 \%$ & 4 & $1.43 \%$ \\
\hline Icononzo & 1 & 2 & $0.72 \%$ \\
\hline
\end{tabular}

\begin{tabular}{|c|c|c|c|c|}
\hline \multicolumn{5}{|c|}{ ODONTOLOGÍA GENERAL } \\
\hline \multirow[t]{2}{*}{ Municipio } & \multicolumn{2}{|c|}{ Sector público } & \multicolumn{2}{|c|}{ Sector privado } \\
\hline & Cantidad & Porcentaje & Cantidad & Porcentaje \\
\hline Líbano & 1 & $1.08 \%$ & 8 & $2.87 \%$ \\
\hline Mariquita & 1 & $1.08 \%$ & 15 & $5.38 \%$ \\
\hline Melgar & 1 & $1.08 \%$ & 7 & $2.51 \%$ \\
\hline Murillo & 1 & $1.08 \%$ & 0 & $0 \%$ \\
\hline Natagaima & 1 & $1.08 \%$ & 2 & $0.72 \%$ \\
\hline Ortega & 1 & $1.08 \%$ & 3 & $1.08 \%$ \\
\hline Palocabildo & 1 & $1.08 \%$ & 0 & $0 \%$ \\
\hline Piedras & 2 & $2.15 \%$ & 0 & $0 \%$ \\
\hline Planadas & 3 & $3.22 \%$ & 3 & $1.08 \%$ \\
\hline Prado & 1 & $1.08 \%$ & 2 & $0.72 \%$ \\
\hline Purificación & 1 & $1.08 \%$ & 2 & $0.72 \%$ \\
\hline Rioblanco & 2 & $2.15 \%$ & 2 & $0.72 \%$ \\
\hline Roncesvalles & 1 & $1.08 \%$ & 0 & $0 \%$ \\
\hline Rovira & 1 & $1.08 \%$ & 1 & $0.36 \%$ \\
\hline Saldaña & 1 & $1.08 \%$ & 2 & $0.72 \%$ \\
\hline San Antonio & 3 & $3.22 \%$ & 1 & $0.36 \%$ \\
\hline San Luis & 1 & $1.08 \%$ & 0 & $0 \%$ \\
\hline Santa Isabel & 1 & $1.08 \%$ & 0 & $0 \%$ \\
\hline Suárez & 1 & $1.08 \%$ & 0 & $0 \%$ \\
\hline Valle de San Juan & 1 & $1.08 \%$ & 0 & $0 \%$ \\
\hline Venadillo & 1 & $1.08 \%$ & 0 & $0 \%$ \\
\hline Villahermosa & 1 & $1.08 \%$ & 0 & $0 \%$ \\
\hline Villarrica & 1 & $1.08 \%$ & 0 & $0 \%$ \\
\hline Total & 101 & $100 \%$ & 279 & $100 \%$ \\
\hline
\end{tabular}

Fuente: Elaborado a partir de los datos del portal electrónico del Ministerio de Salud colombiano. 


\section{Cuadro 3.}

Municipios del departamento del Tolima con requerimiento adicional de odontólogos/as

\begin{tabular}{|c|c|c|c|c|c|}
\hline \multicolumn{6}{|c|}{ MUNICIPIOS CON REQUERIMIENTO ADICIONAL DE ODONTÓLOGOS } \\
\hline Relación ideal & +1 & +2 & +3 & 4 a 10 & $>10$ \\
\hline Alpujarra & Murillo & Alvarado & $\begin{array}{l}\text { Armero- } \\
\text { Guayabal }\end{array}$ & Anzoátegui & Ibagué \\
\hline Casabianca & Roncesvalles & Ambalema & Coello & Ataco & Chaparral \\
\hline Piedras & Santa Isabel & Carmen de Apicalá & Cunday & Cajamarca & Espinal \\
\hline & Suárez & Dolores & Icononzo & Coyaima & Guamo \\
\hline & Valle de San Juan & Falan & Villa Hermosa & Flandes & Líbano \\
\hline & Villa Rica & Herveo & & Fresno & Mariquita \\
\hline & & Palocabildo & & Honda & Melgar \\
\hline & & San Antonio & & Lérida & \\
\hline & & & & Natagaima & \\
\hline & & & & Ortega & \\
\hline & & & & Planadas & \\
\hline & & & & Prado & \\
\hline & & & & Purificación & \\
\hline & & & & Rioblanco & \\
\hline & & & & Rovira & \\
\hline & & & & Saldaña & \\
\hline & & & & San Luis & \\
\hline & & & & Venadillo & \\
\hline
\end{tabular}

Fuente: Elaborado con los cálculos del archivo de Excel, en apego a las recomendaciones de la Organización Mundial de la Salud.

\section{Cuadro 4.}

Tiempo, distancia y velocidad promedio de viaje real a cuatro municipios del departamento del Tolima

\begin{tabular}{|l|r|r|r|}
\hline \multicolumn{4}{|c|}{ MUNICIPIOS CON REQUERIMIENTO ADICIONAL DE ODONTÓLOGOS } \\
\hline Municipio & $\begin{array}{c}\text { Tiempo de recorrido } \\
\text { en horas }\end{array}$ & $\begin{array}{c}\text { Distancia recorrida } \\
\text { en km }\end{array}$ & $\begin{array}{c}\text { Velocidad promedio } \\
\mathrm{km} / \mathrm{h}\end{array}$ \\
\hline Icononzo & 2.75 & 119.4 & 52.5 \\
\hline Herveo & 6.1 & 217.1 & 36.8 \\
\hline Roncesvalles & 6.12 & 119.3 & 17.2 \\
\hline Planadas & 7.1 & 230.5 & 67.7 \\
\hline
\end{tabular}

Fuente: Elaborado con los datos recolectados por la coautora. El resaltado muestra que tres de los cuatro municipios presentan barrera de acceso geográfico. 
Cuadro 5.

Tiempo de viaje hasta Ibagué en bus por carretera pavimentada y afirmada

\begin{tabular}{|c|c|c|c|c|c|c|}
\hline Municipio & $\begin{array}{c}\text { Distancia hasta } \\
\text { Ibagué }\end{array}$ & $\begin{array}{c}\text { Kms } \\
\text { pavimentados }\end{array}$ & $\begin{array}{c}\text { Tiempo de } \\
\text { viaje } 52 \\
\text { Km/H }\end{array}$ & $\begin{array}{c}\text { Kms } \\
\text { afirmados }\end{array}$ & $\begin{array}{c}\text { Tiempo de } \\
\text { viaje } 17 \\
\text { Km/H }\end{array}$ & $\begin{array}{l}\text { Tiempo total } \\
\text { de viaje }\end{array}$ \\
\hline Ibagué & 0 & 0 & 0 & 0 & 0 & 0 \\
\hline Alpujarra & 171 & 160 & 3.07 & 11 & 0.64 & 3.71 \\
\hline Alvarado & 41.8 & 41.8 & 0.80 & 0 & 0 & 0.80 \\
\hline Ambalema & 79 & 79 & 1.52 & 0 & 0 & 1.52 \\
\hline Anzoátegui & 85.2 & 85.2 & 1.64 & 0 & 0 & 1.64 \\
\hline Armero - Guayabal & 99.8 & 99.8 & 1.92 & 0 & 0 & 1.92 \\
\hline Ataco & 154 & 154 & 2.96 & 0 & 0 & 2.96 \\
\hline Cajamarca & 34.2 & 34.2 & 0.66 & 0 & 0 & 0.66 \\
\hline Carmen de Apicalá & 89.5 & 89.5 & 1.72 & 0 & 0 & 1.72 \\
\hline Casabianca & 156 & 133 & 2.56 & 23 & 1.35 & 3.91 \\
\hline Chaparral & 152 & 152 & 2.92 & 0 & 0 & 2.92 \\
\hline Coello & 60.1 & 60.1 & 1.15 & 0 & 0 & 1.15 \\
\hline Coyaima & 116 & 116 & 2.23 & 0 & 0 & 2.23 \\
\hline Cunday & 108 & 108 & 2.02 & 0 & 0 & 2.02 \\
\hline Dolores & 145 & 145 & 2.79 & 0 & 0 & 2.79 \\
\hline El Espinal & 58 & 58 & 1.11 & 0 & 0 & 1.11 \\
\hline Falan & 122 & 122 & 2.35 & 0 & 0 & 2.35 \\
\hline Flandes & 65.2 & 65.2 & 1.25 & 0 & 0 & 1.25 \\
\hline Fresno & 144 & 144 & 2.77 & 0 & 0 & 2.77 \\
\hline El Guamo & 71.5 & 71.5 & 1.38 & 0 & 0 & 1.38 \\
\hline Herveo & 196 & 179.4 & 3.45 & 16.6 & 0.98 & 4.43 \\
\hline Honda & 140 & 140 & 2.69 & 0 & 0 & 2.69 \\
\hline Icononzo & 119 & 119 & 2.29 & 0 & 0 & 2.29 \\
\hline Lérida & 78.8 & 78.8 & 1.52 & 0 & 0 & 1.52 \\
\hline Líbano & 124 & 124 & 2.38 & 0 & 0 & 2.38 \\
\hline Mariquita & 120 & 120 & 2.31 & 0 & 0 & 2.31 \\
\hline Melgar & 87.3 & 87.3 & 1.68 & 0 & 0 & 1.68 \\
\hline Murillo & 147 & 147 & 2.83 & 0 & 0 & 2.82 \\
\hline Natagaima & 121 & 121 & 2.33 & 0 & 0 & 2.33 \\
\hline Ortega & 108 & 108 & 2.08 & 0 & 0 & 2.08 \\
\hline Palocabildo & 133 & 133 & 2.56 & 0 & 0 & 2.56 \\
\hline Piedras & 53 & 53 & 1.02 & 0 & 0 & 1.02 \\
\hline Planadas & 230 & 154 & 2.96 & 76 & 4.47 & 7.43 \\
\hline Prado & 112 & 112 & 2.15 & 0 & 0 & 2.15 \\
\hline Purificación & 99.4 & 85.2 & 1.63 & 14.2 & 0.84 & 2.47 \\
\hline
\end{tabular}




\begin{tabular}{|l|r|r|r|r|r|r|}
\hline \multicolumn{1}{|c|}{ Municipio } & $\begin{array}{c}\text { Distancia hasta } \\
\text { lbagué }\end{array}$ & $\begin{array}{c}\text { Kms } \\
\text { Pavimentados }\end{array}$ & $\begin{array}{c}\text { Tiempo de } \\
\text { viaje } 52 \\
\text { Km/H }\end{array}$ & $\begin{array}{c}\text { Kms } \\
\text { Afirmados }\end{array}$ & $\begin{array}{c}\text { Tiempo de } \\
\text { viaje } 17 \\
\text { Km/H }\end{array}$ & $\begin{array}{c}\text { Tiempo total } \\
\text { de viaje }\end{array}$ \\
\hline Rioblanco & 206 & 152 & 2.92 & 54 & 3.17 & 6.09 \\
\hline Roncesvalles & 114 & 38 & 0.73 & 76 & 4.47 & 5.2 \\
\hline Rovira & 38 & 38 & 0.73 & 0 & 0 & 0.73 \\
\hline Saldaña & 85.2 & 85.2 & 1.64 & 0 & 0 & 1.64 \\
\hline San Antonio & 187 & 152 & 2.92 & 35 & 2.06 & 4.98 \\
\hline San Luis & 62.8 & 62.8 & 1.21 & 0 & 0 & 1.21 \\
\hline Santa Isabel & 99 & 99 & 1.90 & 0 & 0 & 1.90 \\
\hline Suárez & 91.2 & 91.2 & 1.75 & 0 & 0 & 1.75 \\
\hline Valle de San Juan & 46 & 46 & 0.88 & 0 & 0 & 0.88 \\
\hline Venadillo & 59.3 & 59.3 & 1.14 & 0 & 0 & 1.14 \\
\hline Villa Hermosa & 154 & 124 & 2.38 & 30 & 1.76 & 4.14 \\
\hline Villa Rica & 139 & 108 & 2.07 & 31 & 1.82 & 3.89 \\
\hline
\end{tabular}

Fuente: Elaborado con la nueva fórmula empleada en el trabajo. Se sombrean los municipios con barrera de acceso geográfico.

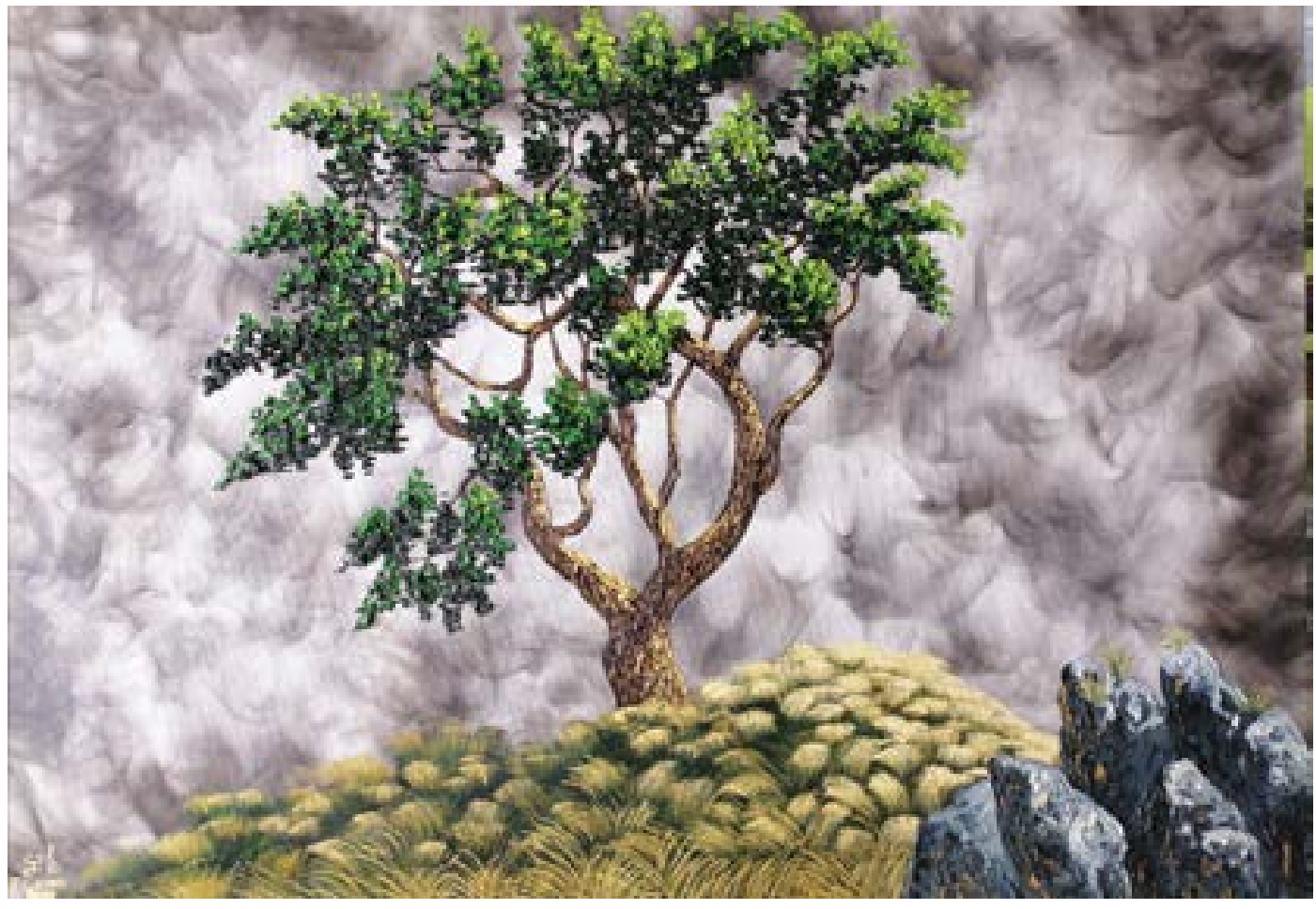

"Yuku ñú'ma"

Humo, óleo sobre tela

$70 \times 100 \mathrm{~cm}$

2020 